\title{
Reposicionamiento labial: reporte de caso
}

\author{
Lip repositioning: case report \\ Reposicionamento labial: relato de caso
}

Recibido: 03/09/2021 | Revisado: 06/09/2021 | Acepto: 06/09/2021 | Publicado: 07/09/2021

\author{
Valentina Martínez Peraza \\ ORCID: https://orcid.org/0000-0002-4033-2590 \\ Universidad de Cuenca, Ecuador \\ E-mail: valentina.martinez@ucuenca.edu.ec \\ Katherine Andrea Romero Espinoza \\ ORCID: https://orcid.org/0000-0002-7843-9676 \\ Universidad de Cuenca, Ecuador \\ E-mail: andrea.romero@ucuenca.edu.ec
}

\begin{abstract}
Resumen
La sonrisa gingival es una condición clínica caracterizada por una exposición gingival mayor a 4mm al sonreír, afectando estéticamente a muchos individuos. La presencia de sonrisa gingival se debe a condiciones etiológicas multifactoriales; es por ello que para su corrección se debe establecer un correcto diagnóstico determinando él o los factores etiológicos para llevar a cabo un efectivo plan de tratamiento. A continuación se presenta el reporte de caso de una mujer de 24 años que presenta amplia exposición gingival al sonreír debida hipermovilidad labial y a erupción pasiva alterada. Por lo tanto, se realizo gingivectomía en combinación con la técnica de reposicionamiento labial, está técnica consiste en eliminar una banda de mucosa del vestíbulo maxilar creando un colgajo de espesor parcial entre la unión de la línea mucogingival y apical a la musculatura del labio superior, para luego suturar y lograr un vestíbulo más corto; restringiendo la musculatura del labio superior dando resolución a la excesiva exposición gingival. Se concluye que ambos procedimientos fueron exitosos, predecibles y poco invasivos obteniendo un resultado satisfactorio sin reportar recidivas en 6 meses.
\end{abstract}

Palabras clave: Sonrisa gingival; Excesiva exposición gingival; Labio hipermóvil; Erupción pasiva alterada; Gingivectomía; Técnica de reposicionamiento labial.

\begin{abstract}
Gummy smile is a clinical condition in which a gingival exposure greater than $4 \mathrm{~mm}$ occurs when smiling, aesthetically affecting many individuals. Gummy smile is due to multifactorial etiological conditions; that is why, for its correction, a correct diagnosis must be established, determining the etiological factor (s) to carry out an effective treatment plan. The following is a case report of a 24-year-old woman who presented extensive gingival exposure when smiling due to a hypermobile lip and an altered passive eruption, for which a gingivectomy was performed in combination with the lip repositioning technique, eliminating a band of mucosa of the maxillary vestibule creating a partial thickness flap between the junction of the mucogingival line and apical to the upper lip musculature to later suture and achieve a shorter vestibule, restricting the upper lip musculature giving resolution to the excessive gingival display. It is concluded that both procedures were successful, predictable, and minimally invasive, obtaining a satisfactory result without reporting relapses in 6 months. Keywords: Gummy smile; Excessive gingival exposure; Hypermobile lip; Altered passive eruption; Gingivectomy; Lip repositioning technique.
\end{abstract}

\footnotetext{
Resumo

O sorriso gengival é uma condição clínica em que ao sorrir ocorre uma exposição gengival maior que 4mm, afetando esteticamente muitos indivíduos. A presença do sorriso gengival deve-se a condições etiológicas multifatoriais; por isso, para sua correção, deve-se estabelecer um diagnóstico correto, determinando o (s) fator (es) etiológico (s) para a realização de um plano de tratamento eficaz. A seguir está o relato de um caso de uma mulher de 24 anos que apresentou extensa exposição gengival ao sorrir devido a um lábio hipermóvel e uma erupção passiva alterada, para a qual foi realizada gengivectomia em combinação com a técnica de reposicionamento labial, eliminando uma faixa de mucosa do vestíbulo maxilar criando um retalho de espessura parcial entre a junção da linha mucogengival apical à musculatura do lábio superior para suturar posteriormente e atingir um vestíbulo mais curto, restringindo a musculatura do lábio superior dando resolução
} 
ao sorriso gengival. Conclui-se que ambos os procedimentos foram bem-sucedidos, previsíveis e minimamente invasivos, obtendo um resultado satisfatório sem relatar recidivas em 6 meses.

Palavras-chave: Sorriso gengival; Exposição gengival excessiva; Lábio hipermóvel; Erupção passiva alterada; Gengivectomia; Técnica de reposicionamento labial.

\section{Introducción}

La sonrisa siempre ha sido considerada como una característica determinante a tomar en cuenta en la estética de un individuo. El sonreír implica una combinación entre las contracciones musculares, la posición del labio, las características de la mucosa bucal, la encía y los dientes. Es por ello que muchas veces, cuando alguna de estas estructuras es afectada por problemas en el desarrollo o por algunas condiciones adquiridas, los individuos refieren una disconformidad estética con su sonrisa. (dos SantosPereira et al., 2021), (Alammar, Heshmeh, Mounajjed, Goodson, \& Hamadah, 2018; Bhimani \& Sofia, 2019; Tawfik, El-Nahass, et al., 2018)

Entre estas afecciones la amplia exposición gingival al sonreir, conocido como "sonrisa gingival”, definida como una condición no patológica en donde la exposición gingival supera los 4mm tomados desde el extremo inferior del labio superior y el margen gingival de los dientes, razón por la que muchos pacientes refieren malestar estético y buscan soluciones en tratamientos odontológicos. (Dym \& Pierre 2nd, 2020; Sheth, Shah, Shah, \& Shah, 2013)

La incidencia de la excesiva exposición gingival es debida a condiciones etiológicas multifactoriales como: el labio superior corto o hiperactivo, erupción pasiva alterada y condiciones esqueletales como el exceso vertical de hueso maxilar. En cuanto a su prevalencia se ha reportado que las mujeres presentan mayor predisposición que los hombres en una relación de 14\% a 7\% respectivamente. (Smile, 2014)

La terapéutica de la sonrisa gingival es variada y puede ser manejado de diferentes formas de acuerdo con el factor etiológico que se presente; la resolución del caso puede ir desde tratamientos estéticos, procedimientos restaurativos y cirugía plástica periodontal hasta casos más complejos como cirugía ortognática, inyecciones de toxina botulínica, cirugía de reposicionamiento labial, o la combinación de alguna de éstas. (Aroni, Pigossi, Pichotano, de Oliveira, \& Marcantonio, 2019; Chagas et al., 2018; Paik, Park, \& Ahn, 2017; Tawfik, El-Nahass, et al., 2018; Tawfik, Naiem, et al., 2018)

La cirugía de reposicionamiento labial es una herramienta útil cuando la etiología está asociada a hipermovilidad labial, que según las subclases definidas por Monish Bhola en el año 2015 podrá combinarse con la aplicación de toxina botulínica tipo A. La cirugía de reposicionamiento labial además se puede combinar con otros procedimientos como alargamiento de corona clínica estético, intrusión ortodóntica, cirugía ortognática y ejercicios de entrenamiento labial cuando existan otros factores etiológicos asociados a la sonrisa gingival. La técnica fue presentada por primera vez en 1973 en una revista de cirugía plástica por Rubinstein y Kostianosvsky. procedimiento que ha presentado diferentes modificaciones a través de los años, sin embargo, el objetivo a lograr con esta cirugía es limitar el movimiento de los músculos elevadores al sonreir, así como acortar el vestíbulo. La técnica quirúrgica se realiza removiendo una banda mucosa basada en la “regla de dos veces la suma de encía expuesta”. (Alammar et al., 2018; Bhola et al., 2015; Dilaver \& Uckan, 2018; Mann, 2017; Silva, Ribeiro-Júnior, Campos, Rodrigues, \& Tatakis, 2013)

Para tratar la sonrisa gingival la técnica de reposicionamiento labial ha mostrado resultados favorables a corto plazo, sin embargo, es una cirugía muy controversial ya que se han reportado algunas recidivas. (Dilaver \& Uckan, 2018; Ishida et al., 2010; Ozturan, Ay, \& Sagir, 2014)

Por consiguiente, el propósito de este reporte de caso es mostrar el empleo de la técnica de reposicionamiento labial para tratar y rectificar la sonrisa gingival y obtener los parámetros estéticos requeridos por el paciente. 


\section{Metodología}

Este reporte de caso se llevó a cabo con enfoque metodológico cualitativo descriptivo. Para su desarrollo no se requirió la aplicación de encuestas, puesto que se contó con una detallada historia y análisis clínico minucioso de los que se obtuvieron la información necesaria para el diagnóstico, plan de tratamiento, y ejecución terapéutica del caso. La publicación cumple con los principios éticos, puesto que, para llevar a cabo este reporte, el paciente firmó el consentimiento informado de la intervención quirúrgica, así como la aprobación para su publicación; en todo momento se respeta el anonimato del paciente. (Pereira, Shitsuka, Parreira, \& Shitsuka, 2018)

Los autores se autofinancian y tienen el apoyo docente de la Facultad de Odontología de la Universidad de Cuenca.

\subsection{Reporte de caso}

Paciente femenina de 24 años, acudió a consulta periodontal manifestando disconformidad estética por exposición gingival excesiva al sonreír. En la anamnesis la paciente no refiere antecedentes sistémicos. Al examen clínico se observa salud periodontal y una moderada exposición gingival al sonreír. (Figura 1)

Se realizó una examinación extraoral e intraoral detallada. En cuanto a las medidas extraorales se consideró los 3 tercios de la cara, superior, medio e inferior, además se registraron medidas para identificar la extensión vertical y horizontal de la sonrisa tanto en reposo como en movimiento. En el análisis intraoral fueron registradas dimensiones del largo y ancho de las coronas clínicas dentales determinando zenit gingivales bajos de canino a canino, adicionalmente se midió la encía queratinizada presente y la localización de la línea mucogingival. Radiográficamente se midió la relación entre el límite amelocementario de los dientes y la altura de la cresta alveolar. Con los datos obtenidos se constató que los factores etiológicos de la sonrisa gingival de la paciente estaban relacionados con hipermovilidad labial y erupción pasiva alterada.

Figura 1. Imagen clínica de la exposición gingival al sonreir. Se observa la excesiva exposición gingival.

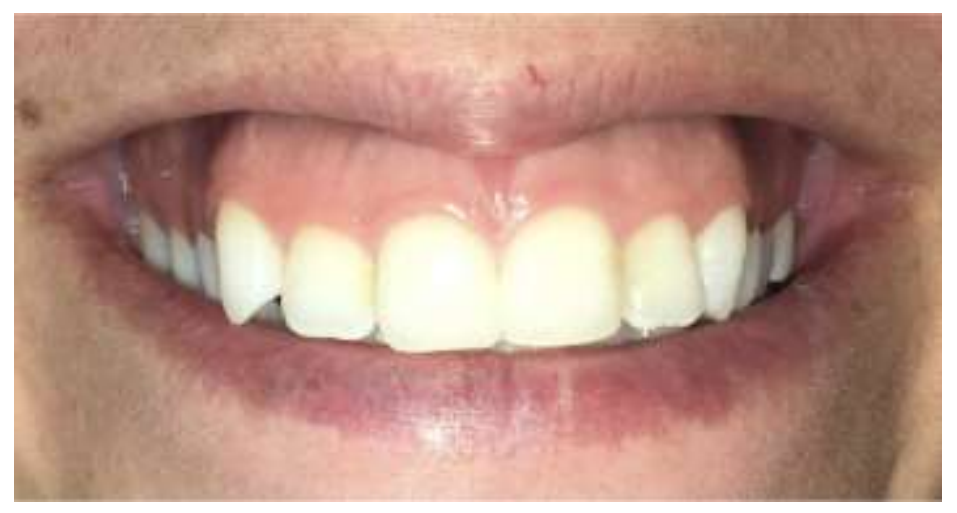

Fuente Od. Katherine Romero.

Para remediar la sonrisa gingival se realizó la técnica quirúrgica de reposicionamiento labial conjuntamente con una gingivectomía. Las cirugías se realizaron con anestesia infiltrativa en el maxilar de segundo premolar derecho a segundo premolar izquierdo con lidocaína al 2\% con 1:100,000 de epinefrina. El primer procedimiento realizado fue el de gingivectomía para aumentar el largo de las coronas de los dientes anterosuperiores con hoja de bisturí número 15c. (Figura 2) 
Una vez finalizada la gingivectomía se procedió a realizar la cirugía de reposicionamiento labial, dibujando el diseño del colgajo con un lápiz quirúrgico (Figura 2). Se realizó la primera incisión para un colgajo de espesor parcial siguiendo la unión de la línea mucogingival extendiéndose de segundo premolar derecho a segundo premolar izquierdo que fue el resultado de la medida de la sonrisa dinámica, acto seguido se realizó la segunda incisión a 10mm apical a la línea mucogingival siguiendo la "regla de dos veces la suma de encía expuesta“. Se removió el epitelio cuidadosamente aproximadamente $1 \mathrm{~mm}$ hasta exponer el tejido conectivo dejando el músculo y las fibras intactas. Se aplicó presión para lograr hemostasia. Luego los márgenes del colgajo fueron aproximados con puntos simples empezando por el centro y luego a los lados guardando la misma distancia entre ellos utilizando sutura no reabsorbible Nylon 6-0. (Figura 3)

Figura 2. Imagen clínica de gingivectomía realizada de segundo premolar izquierdo a segundo premolar derecho así como del diseño del colgajo con lápiz quirúrgico para realizar el reposicionamiento labial.

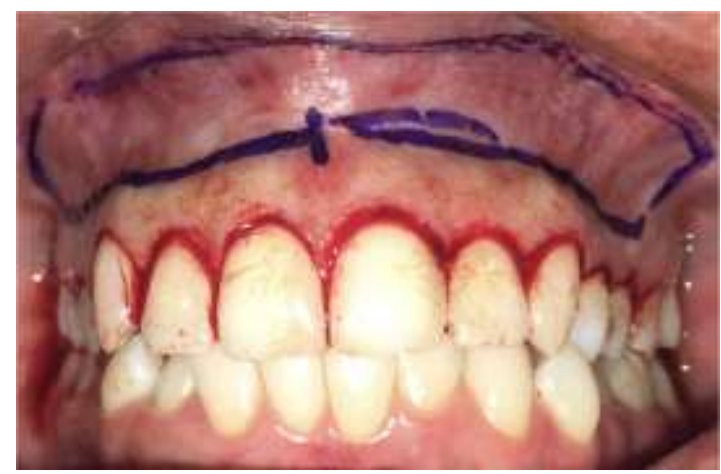

Fuente Od. Katherine Romero.

Figura 3. Imagen clínica del lecho quirúrgico suturado. Se observa simetría entre lado derecho e izquierdo y disminución del ancho del vestíbulo.

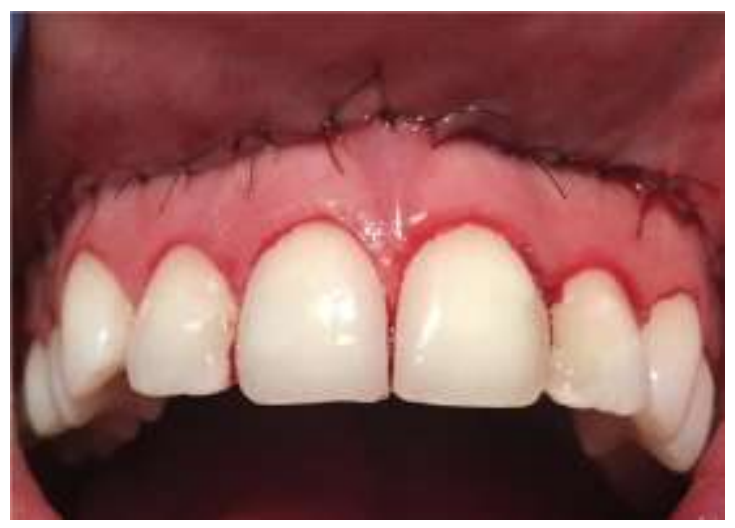

Fuente Od. Katherine Romero.

Fueron prescritos analgésicos y antiinflamatorios como medicamentos posoperatorios combinado con la aplicación de frio a intervalos en la zona intervenida, además la paciente fue instruida con cuidados para limitar la movilización del labio al conversar o sonreír, dieta adecuada y enjuagues con Clorhexidina al 12\% 10ml 2 veces al día por 14 días. Se efectuaron controles posquirúrgicos a los 7 y 14 días, observando una evaluación adecuada del procedimiento, para finalmente remover la sutura. (Figura 4) 
Figura 4. Imagen clínica de control posoperatorio con sutura a los 15 días. Se observa la mejoría en la en la exposición al sonreir.

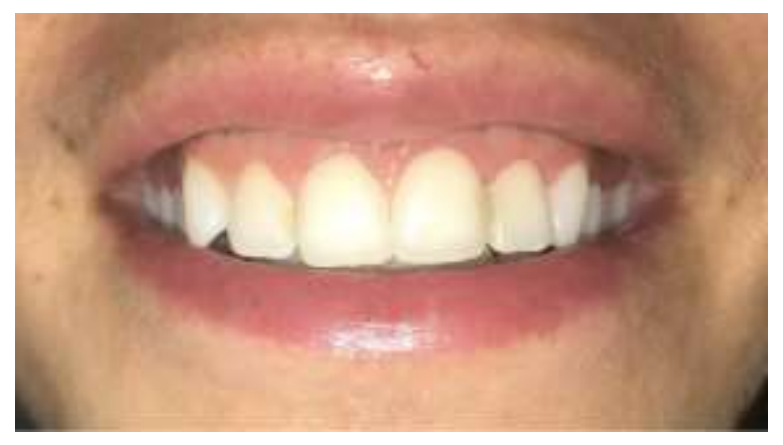

Fuente Od. Katherine Romero.

Se realizó un control al mes y 6 meses, identificándose estabilidad en el tiempo evaluado, manifestando la paciente satisfacción por la resolución lograda. (Figura 5)

Figura 5. Imagen clínica de control a los 6 meses. Completa resolución estética de la sonrisa gingival.

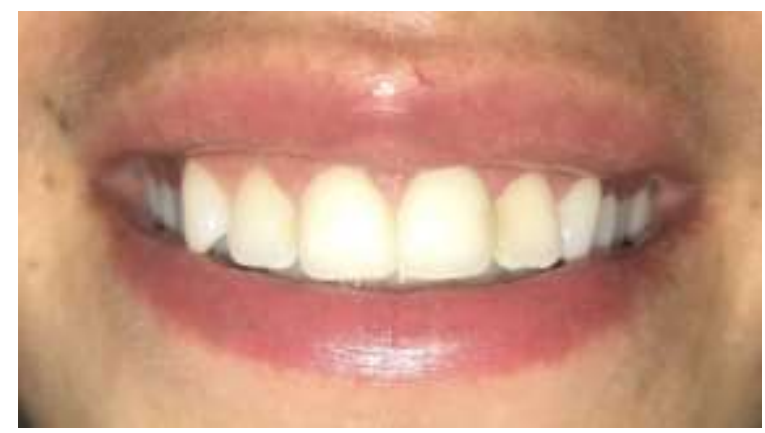

Fuente Od. Katherine Romero.

\section{Resultados y Discusión}

En este reporte de caso se realizó la técnica quirúrgica de reposicionamiento labial en combinación con alargue coronal estético como herramienta para solucionar la sonrisa gingival luego de identificar los agentes causales asociados (hipermovilidad labial y erupción pasiva alterada).

Esta técnica quirúrgica fue explicada en el año de 1973 por primera vez por Rubinstein y Kostianovsky para tratar la sonrisa gingival ocasionada por hipermovilidad labial. Más adelante Litton y Fournier (1979) realizaron reposicionamientos labiales para corregir casos clínicos de labio superior corto; en esta ocasión la técnica separa los músculos del hueso para posicionar coronalmente el labio superior. (Litton \& Fournier, 1979; Rubinstein \& Kostianovsky, 1973)

Después de 25 años otros autores practicaron la técnica utilizando una incisión de espesor parcial de forma elíptica en la mucosa alveolar, removiendo de 10 a $12 \mathrm{~mm}$ de epitelio, con la finalidad de mantener las fibras y los músculos intactos, buscando resultados sin recidivas al cabo de 8 meses. (Rosenblatt \& Simon, 2006; Simon, Rosenblatt, \& Dorfman, 2007)

Más adelante Humayun (2010) reportó un caso donde se posicionó el colgajo hacia coronal en la mucosa para tratar el exceso vertical del maxilar y el labio hipermóvil y así disminuir la excesiva exposición gingival; este caso fue el primero en aplicar la regla de "dos veces la suma de encía expuesta" logrando estabilizar los resultados sin recidivas por 1 año. (Humayun, Kolhatkar, Souiyas, \& Bhola, 2010). 
En el mismo año Ishida (2010) describe una técnica quirúrgica en la que se realiza miotomía del músculo elevador del labio superior, disección perióstica de la encía, disección subcutánea del labio y frenectomía para corregir la excesiva exposición gingival, aún así, la técnica mostró mayor morbilidad en el posoperatorio y en algunos casos se reportó parestesia del labio superior. (Ishida et al., 2010)

El estudio más importante reportado sobre reposicionamiento labial fue realizado por Silva et al., en la cual se mantuvo la inserción del frenillo labial superior preservando así la posición de la línea media, evitando la morbilidad que se había reportó años atrás. Posteriormente, Bhola et al., publicaron una clasificación sobre causas y tratamientos para la sonrisa gingival, estudio que actualmente es una guía práctica del procedimiento. (Bhola et al., 2015; Silva et al., 2013)

Semejante al caso reportado en el presente artículo, Asha Ramesh (2019) realizó un alargue coronario estético con gingivectomía seguida de la técnica de reposicionamiento labial. La técnica indica una incisión horizontal de segundo premolar izquierdo a segundo premolar derecho en el límite de la unión mucogingival y paralela a esta una segunda incisión 10-12mm en sentido apical, concluyendo con la unión de las dos incisiones horizontales; luego la eliminación cuidadosa del epitelio conservando el tejido conectivo, las fibras y el músculo intacto. Tras el reporte de un posoperatorio exitoso, el resultado se mantuvo intacto en las supervisiones realizadas en controles cada 3 meses sin presentar recidivas por 2 años. (Ramesh, Vellayappan, Ravi, \& Gurumoorthy, 2019)

Es importante destacar que la sonrisa gingival puede tener múltiples causales, la misma que debe ser tratada acorde a un análisis minucioso y un diagnóstico preciso que permita orientar un tratamiento correcto apegado a las necesidades del paciente. (Foudah, 2019)

La técnica de reposicionamiento labial está sugerida para casos de exceso vertical maxilar grado I (2-4mm) y moderado o grado II (4-8mm) de exposición gingival; al igual que en casos de presentar hipermovilidad labial.(Bhola et al., 2015; Humayun et al., 2010)

Por otra parte, es una técnica contraindicada en cuadros clínicos de exceso vertical maxilar grado III (mayor a 8mm) de exposición gingival, poca cantidad de encía queratinizada y vestíbulo corto. (Bhola et al., 2015; Rosenblatt \& Simon, 2006)

En cuanto a las complicaciones posoperatorias reportadas se han identificado: dolor, inflamación, hematomas, mucoceles, y complicaciones atípicas como: daño en las glándulas salivales menores que se resuelven por sí mismas con el tiempo. (Foudah, 2019; Pandurić, Blašković, Brozović, \& Sušić, 2014)

Finalmente, al respecto de esta técnica se debe mencionar que pueden ocurrir recidivas a lo largo del tiempo; la recidiva puede deberse a distintos factores, entre éstos el más aceptado es la memoria muscular. Otros casos a los cuales se les atribuye la recidiva son: no acatar la regla de "dos veces la suma de encía expuesta" al realizar las incisiones, incidir sobre encía queratinizada, incisiones profundas que afecte el tejido conectivo y fibras musculares, o, en ocasiones demasiada tensión muscular. Aún así , varios autores sugieren el empleo de la técnica de reposicionamiento labial en combinación con otras técnicas quirúrgicas y no quirúrgicas para lograr óptimos resultados a largo plazo, ya que se reporta como una técnica poco invasiva, segura y predecible para la resolución de la sonrisa gingival siempre que se cuente con un diagnóstico correcto. (Alammar et al., 2018; dos Santos-Pereira et al., 2021; Foudah, 2019)

Con estas consideraciones se puede mencionar otros tratamientos menos invasivos y no quirúrgico como: la aplicación de toxina botulínica tipo A utilizado con el fin de reducir la exposición gingival excesiva al sonreir y disminuir la hipermovilidad labial causado por los músculos elevadores del labio superior. Sin embargo, para obtener éxito prolongado con esta técnica, se deben realizar varias aplicaciones. (Bhola et al., 2015; Polo, 2008) 


\section{Conclusiones}

Hoy en día, donde los parámetros estéticos de la sonrisa han alcanzado un alto impacto, el presentar una exposición gingival excesiva al sonreir puede ser considerado como un factor que afecta la estabilidad emocional y social de los pacientes y que requiere tratamientos adecuados.

La sonrisa gingival es una condición multifactorial, situación que obliga a realizar una evaluación correcta para determinar su etiología y plantear estrategias adecuadas con un abordaje correcto en su plan de tratamiento. La técnica de reposicionamiento labial es considerada una opción poco invasiva, de buen pronóstico, corta recuperación y con pocas complicaciones posoperatorias para tratar casos específicos de excesiva exposición gingival. Aún así, se puede considerar combinar la técnica de reposicionamiento labial con otros tratamientos quirúrgicos y no quirúrgicos para lograr un resultado más adecuado, predecible y estable, sin dejar a un lado que aún es una técnica que reporta recidivas, siendo un factor importante para tomar en cuenta con el paciente.

Son necesarios nuevos avances clínicos con muestras más numerosas de pacientes y controles posoperatorios a largo plazo para corroborar la efectividad de la técnica quirúrgica.

\section{Referencias}

Alammar, A., Heshmeh, O., Mounajjed, R., Goodson, M., \& Hamadah, O. (2018). A comparison between modified and conventional surgical techniques for surgical lip repositioning in the management of the gummy smile. Journal of Esthetic and Restorative Dentistry, 30(6), 523-531.

Aroni, M. A. T., Pigossi, S. C., Pichotano, E. C., de Oliveira, G., \& Marcantonio, R. A. C. (2019). Esthetic crown lengthening in the treatment of gummy smile. Int J Esthet Dent, 14(4), 370-382.

Bhimani, R. A., \& Sofia, N. D. (2019). Lip repositioning, aesthetic crown lengthening, and gingival depigmentation: A combined approach for a gummy smile makeover. Journal of cutaneous and aesthetic surgery, 12(4), 240.

Bhola, M., Fairbairn, P. J., Kolhatkar, S., Chu, S. J., Morris, T., \& de Campos, M. (2015). LipStaT: The Lip Stabilization Technique—-Indications and Guidelines for Case Selection and Classification of Excessive Gingival Display. International Journal of Periodontics \& Restorative Dentistry, 35(4).

Chagas, T. F., Almeida, N. V. d., Lisboa, C. O., Ferreira, D. M. T. P., Mattos, C. T., \& Mucha, J. N. (2018). Duration of effectiveness of Botulinum toxin type A in excessive gingival display: a systematic review and meta-analysis. Brazilian oral research, 32.

Dilaver, E., \& Uckan, S. (2018). Effect of V-Y plasty on lip lengthening and treatment of gummy smile. International journal of oral and maxillofacial surgery, 47(2), 184-187.

Dos Santos-Pereira, S. A., Cicareli, Á. J., Idalgo, F. A., Nunes, A. G., Kassis, E. N., Castanha Henriques, J. F., \& Bellini-Pereira, S. A. (2021). Effectiveness of lip repositioning surgeries in the treatment of excessive gingival display: A systematic review and meta-analysis. Journal of Esthetic and Restorative Dentistry, 33(3), 446-457.

Dym, H., \& Pierre 2nd, R. (2020). Diagnosis and treatment approaches to a" Gummy Smile. Dent Clin North Am, 64(2), 341-349.

Foudah, M. A. (2019). Lip repositioning: An alternative to invasive surgery a 4 year follow up case report. The Saudi dental journal, 31 , S78-S84.

Humayun, N., Kolhatkar, S., Souiyas, J., \& Bhola, M. (2010). Mucosal coronally positioned flap for the management of excessive gingival display in the presence of hypermobility of the upper lip and vertical maxillary excess: A case report. Journal of periodontology, 81(12), 1858-1863.

Ishida, L. H., Ishida, L. C., Ishida, J., Grynglas, J., Alonso, N., \& Ferreira, M. C. (2010). Myotomy of the levator labii superioris muscle and lip repositioning: a combined approach for the correction of gummy smile. Plastic and reconstructive surgery, 126(3), 1014-1019.

Litton, C., \& Fournier, P. (1979). Simple surgical correction of the gummy smile. Plastic and reconstructive surgery, 63(3), 372-373.

Mann, D. (2017). Lip repositioning to eliminate the gummy smile. Inside Dent, 13, 3.

Ozturan, S., Ay, E., \& Sagir, S. (2014). Case series of laser-assisted treatment of excessive gingival display: an alternative treatment. Photomedicine and laser surgery, 32(9), 517-523.

Paik, C.-H., Park, H.-S., \& Ahn, H.-W. (2017). Treatment of vertical maxillary excess without open bite in a skeletal Class II hyperdivergent patient. The Angle Orthodontist, 87(4), 625-633. 
Research, Society and Development, v. 10, n. 11, e502101120218, 2021

(CC BY 4.0) | ISSN 2525-3409 | DOI: http://dx.doi.org/10.33448/rsd-v10i11.20218

Pandurić, D. G., Blašković, M., Brozović, J., \& Sušić, M. (2014). Surgical treatment of excessive gingival display using lip repositioning technique and laser gingivectomy as an alternative to orthognathic surgery. Journal of Oral and Maxillofacial Surgery, 72(2), 404. e401-404. e411.

Pereira, A. S., Shitsuka, D. M., Parreira, F. J., \& Shitsuka, R. (2018). Metodologia da pesquisa científica.

Polo, M. (2008). Botulinum toxin type A (Botox) for the neuromuscular correction of excessive gingival display on smiling (gummy smile). American journal of orthodontics and dentofacial orthopedics, 133(2), 195-203.

Ramesh, A., Vellayappan, R., Ravi, S., \& Gurumoorthy, K. (2019). Esthetic lip repositioning: A cosmetic approach for correction of gummy smile-A case series. Journal of Indian Society of Periodontology, 23(3), 290.

Rosenblatt, A., \& Simon, Z. (2006). Lip repositioning for reduction of excessive gingival display: a clinical report. International Journal of Periodontics \& Restorative Dentistry, 26(5).

Rubinstein, A., \& Kostianovsky, A. (1973). Cosmetic surgery for the malformation of the laugh: original technique. Prensa Med Argent, $60,952$.

Sheth, T., Shah, S., Shah, M., \& Shah, E. (2013). Lip reposition surgery: A new call in periodontics. Contemporary clinical dentistry, 4 (3), 378.

Silva, C. O., Ribeiro-Júnior, N. V., Campos, T. V., Rodrigues, J. G., \& Tatakis, D. N. (2013). Excessive gingival display: treatment by a modified lip repositioning technique. Journal of clinical periodontology, 40(3), 260-265.

Simon, Z., Rosenblatt, A., \& Dorfman, W. (2007). Eliminating a gummy smile with surgical lip repositioning. The journal of cosmetic dentistry, 23(1).

Smile, M. G. (2014). Modifying gummy smile: a minimally invasive approach. The journal of contemporary dental practice, 15(6), 821-826.

Tawfik, O. K., El-Nahass, H. E., Shipman, P., Looney, S. W., Cutler, C. W., \& Brunner, M. (2018). Lip repositioning for the treatment of excess gingival display: A systematic review. Journal of Esthetic and Restorative Dentistry, 30(2), 101-112.

Tawfik, O. K., Naiem, S. N., Tawfik, L. K., Yussif, N., Meghil, M. M., Cutler, C. W., \& El-Nahass, H. E. (2018). Lip repositioning with or without myotomy: A randomized clinical trial. Journal of periodontology, 89(7), 815-823. 